\title{
RELATIONS BETWEEN DOMAINS OF HOLOMORPHY AND MULTIPLE COUSIN'S PROBLEMS
}

\author{
BY JOJT K $\Lambda$ JIW $\Lambda$ R $\Lambda$
}

\section{Introduction.}

Oka [12] proved that a domain $D$ of holomorphy in $C^{n}$ is a Cousin-I domain, that is, any additive Cousin's distribution in $D$ has a solution. On the other hand from Cartan [5]-Behnke-Stein [2]'s theorem, a Cousin-I domain in $C^{2}$ is a domain of holomorphy. In this way any domain of holomorphy in $C^{2}$ can be completely characterized by additive Cousin's problems. For $n \geqq 3$, however, Cartan [6] showed that a Cousin-I domain in $C^{n}$ is not necessarily a domain of holomorphy. In the previous paper [10] we tried to characterize a domain of holomorphy in a Stein manifold by additive Cousin's problems. An open set $G$ in $C^{n}$ is called regular if $G \cap P$ is a Cousin-I open set for any relatively compact polycylinder $P$ in $C^{n}$. We proved that a domain in $C^{n}$ is a domain of holomorphy if and only if it can be exhausted by regular domains. Moreover, we proved that a regular open set is pseudoconvex in the Cartan's sense at its continuous boundary point. Making use of the results of Oka [13] or Docquier-Grauert [7] respectively, we proved that a domain in $C^{n}$ or more generally in a Stein manifold with a smooth boundary is a domain of holomorphy if and only if it is locally regular at its each boundary point.

Concerning multiple Cousin's problems the situation is more or less different. Thullen [16] gave an example of a domain in $C^{2}$ which is not a domain of holomorphy but a Cousin-II domain, that is, a domain in which any multiple

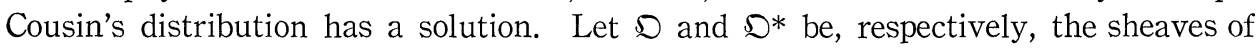
all germs of holomorphic mappings in $C$ and $\mathrm{GL}(1, C)$. As we remarked in [9], Thullen's example is a Cousin-II domain $D$ with $\mathrm{H}^{1}\left(D, D^{*}\right) \neq 0$. In the previous paper [11] we proved that a domain $(D, \varphi)$ over $C^{n}$ with $\mathrm{H}^{1}\left(D, \mathfrak{D}^{*}\right)=\mathrm{H}^{1}\left(\varphi^{-1}(H), \mathfrak{D}^{*}\right)=0$ for any analytic plane $H$ in $C^{n}$ is a domain of holomorphy. Especially a domain $(D, \varphi)$ over $C^{2}$ satisfies $\mathrm{H}^{1}\left(D, D^{*}\right)=0$ if and only if $(D, \varphi)$ is a domain of holomorphy with $\mathrm{H}^{2}(D, Z)=0$ where $Z$ is the abelian group of all integers. These facts suggest that we should obtain a sufficient condition that a domain $D$ in $C^{n}$ is a domain of holomorphy, if we put a similar discussion forward as in [10] substituting a domain $G$ with $\mathrm{H}^{1}\left(G, \mathfrak{D}^{*}\right)=0$ in stead of a Cousin-I domain.

As a polycylinder $P$ does not necessarily satisfy $\mathrm{H}^{1}\left(P, \mathfrak{D}^{*}\right)=0$, we shall consider only simply connected polycylinders in the definition below. An open set $G$ in $C^{n}$ is called regular* if $\mathrm{H}^{1}\left(G \cap P, \mathfrak{D}^{*}\right)=0$ for any relatively compact and simply connected polycylinder $P$ in $C^{n}$. In the present paper we shall prove that a domain

Received March 16, 1965. 
in $C^{n}$ which can be exhausted by regular* domains is a domain of holomorphy and that a regular* domain in $C^{n}$ is pseudoconvex in the Cartan's sense at its continuous boundary point. Making use of the affirmative solution of the Levi problem loco citato, we can prove that a domain over a Stein manifold with a simultaneously continuous and locally regular* boundary is a domain of holomorphy.

\section{$\S 1$. Limit of cohomology groups.}

A sequence $\left\{\left(D_{n}, \varphi_{n}\right) ; n=1,2,3, \cdots\right\}$ of domains $\left(D_{n}, \varphi_{n}\right)$ over a Stein manifold $S$ is called a monotonously increasing sequence of domains over $S$ if there exists a holomorphic mapping $\tau_{m}^{n}$ of $D_{n}$ in $D_{m}$ with $\varphi_{n}=\varphi_{m} \circ \tau_{m}^{n}$ for $m$ and $n$ with $m \geqq n$. In the previous paper [8] we proved the existence of a domain $(D, \varphi)$ over $S$ with the following properties:

(1) There exists a holomorphic mapping $\tau_{n}$ of $D_{n}$ in $D$ with $\varphi_{n}=\varphi \circ \tau_{n}$ for any $n$.

(2) Let $\left(D^{\prime}, \varphi^{\prime}\right)$ be a domain over $S$ such that there exists a holomorphic mapping $\tau_{n}^{\prime}$ of $D_{n}$ in $D^{\prime}$ with $\varphi_{n}=\varphi^{\prime} \circ \tau_{n}^{\prime}$ for any $n$. Then there exists a holomorphic mapping $\tau^{\prime}$ of $D$ in $D^{\prime}$ with $\tau_{n}^{\prime}=\tau^{\prime} \circ \tau_{n}$ for any $n$.

$(D, \varphi)$ is called the limit of the sequence $\left\{\left(D_{n}, \varphi_{n}\right) ; n=1,2,3, \cdots\right\}$. We consider the universal covering manifold $\left(D^{\sharp}, \varphi^{\sharp}\right)$ of $(D, \varphi)$. Let $\lambda$ be the canonical mapping of $D^{*}$ in $D$. Then $\tau_{m}^{n}, \tau_{n}$ and $\lambda$ induce canonically homomorphisms $\tau_{m}^{n *}: \mathrm{H}^{1}\left(D_{m}, \mathbb{D}^{*}\right)$ $\rightarrow \mathrm{H}^{1}\left(D_{n}, \mathfrak{D}^{*}\right), \tau_{n}^{*}: \mathrm{H}^{1}\left(D, \mathfrak{D}^{*}\right) \rightarrow \mathrm{H}^{1}\left(D_{n}, \mathfrak{D}^{*}\right)$ and $\lambda^{*}: \mathrm{H}^{1}\left(D, D^{*}\right) \rightarrow \mathrm{H}^{1}\left(D^{*}, \mathfrak{D}^{*}\right)$. For the sake

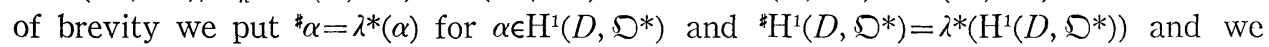
shall use these notations frequently. $\left\{\mathrm{H}^{1}\left(D_{n}, \searrow^{*}\right), \tau_{m}^{n *}\right\}$ is an inverse system of abelian groups over a directed set $\{n=1,2,3, \cdots\}$. We consider its inverse limit and denote it by $\lim \mathrm{H}^{1}\left(D_{n}, \mathfrak{D}^{*}\right)$. We denote the canonical homomorphism of $\mathrm{H}^{1}\left(D, D^{*}\right)$ in $\lim \mathrm{H}^{1}\left(D_{n}, \searrow^{*}\right)$ by $\pi$. Unfortunately we can not yet succeed to prove that $\pi$ is injective but we have the following lemma, which is sufficient for our purpose and the proof of which is quite similar to that of Proposition 2 in the previous paper [8].

Lemma 1. $\alpha \in \mathrm{H}^{1}\left(D, \mathfrak{D}^{*}\right)$ with $\pi(\alpha)=0$ satisfies ${ }^{*} \alpha=0$.

Proof. Let $\left(\widetilde{D}_{n}, \tilde{\varphi}_{n}\right)$ and $(\tilde{D}, \tilde{\varphi})$ be, respectively, the envelopes of holomorphy of $\left(D_{n}, \varphi_{n}\right)$ and $(D, \varphi)$. Let $\left(D_{n}^{\sharp}, \varphi_{n}^{\sharp}\right),\left(D^{\sharp}, \varphi^{\sharp}\right),\left(\tilde{D}_{n}^{\sharp}, \tilde{\varphi}_{n}^{\sharp}\right)$ and $\left(\tilde{D}^{\sharp}, \tilde{\varphi}^{\sharp}\right)$ be, respectively, the universal covering manifolds of $\left(D_{n}, \varphi_{n}\right),(D, \varphi),\left(\tilde{D}_{n}, \tilde{\varphi}_{n}\right)$ and $(\tilde{D}, \tilde{\varphi})$. Since $\left(\widetilde{D}_{n}^{\sharp}, \tilde{\varphi}_{n}^{\sharp}\right)$ and $\left(\tilde{D}^{\sharp}, \tilde{\varphi}^{\sharp}\right)$ are $\mathrm{p}_{7}$-convex in the sense of Docquier-Grauert [7], they are domains of holomorphy from [7]. We consider canonical mappings $\tau_{m}^{n}$ : $D_{n} \rightarrow D_{m}, \tau_{m}: D_{m} \rightarrow D$, $\tau_{m}^{\sharp n}: D_{n}^{*} \rightarrow D_{m}^{*}, \tau_{m}^{*}: D_{m}^{*} \rightarrow D^{\sharp}, \tilde{\tau}_{m}^{\sharp n}: \widetilde{D}_{n}^{*} \rightarrow \widetilde{D}_{m}^{*}, \tilde{\tau}_{m}^{\sharp}: \widetilde{D}_{m}^{*} \rightarrow \widetilde{D}^{\sharp}, \lambda_{n}: D_{n}^{*} \rightarrow D_{n}, \lambda: D^{\sharp} \rightarrow D, \mu_{n}: D_{n} \rightarrow \widetilde{D}_{n}$, $\mu: D \rightarrow \widetilde{D}, \tilde{\lambda}_{n}: \widetilde{D}_{n}^{\sharp} \rightarrow \widetilde{D}_{n}, \tilde{\lambda}: \widetilde{D}^{\sharp} \rightarrow \widetilde{D}, \kappa_{n}: D_{n}^{\sharp} \rightarrow \widetilde{D}_{n}^{\ddagger}$ and $\kappa: D^{\sharp} \rightarrow \tilde{D}^{\ddagger}$ for $1 \leqq n \leqq m$. Then the commutativity holds in the following diagram:

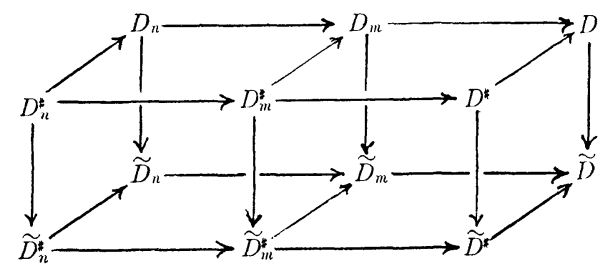


$(D, \varphi),\left(D^{\sharp}, \varphi^{\sharp}\right),(\tilde{D}, \tilde{\varphi})$ and $\left(\tilde{D}^{\sharp}, \tilde{\varphi}^{\sharp}\right)$ are, respectively, limits of monotonously increasing sequences $\left\{\left(D_{n}, \varphi_{n}\right) ; n=1,2,3, \cdots\right\},\left\{\left(D_{n}^{*}, \varphi_{n}^{*}\right) ; n=1,2,3, \cdots\right\},\left\{\left(\widetilde{D}_{n}, \tilde{\varphi}_{n}\right) ; n=1,2,3, \cdots\right\}$ and $\left\{\left(\widetilde{D}_{n}^{\sharp}, \tilde{\varphi}_{n}^{\sharp}\right) ; n=1,2,3, \cdots\right\}$ of domains over $S$. Let $\left\{P_{n} ; n=1,2,3, \cdots\right\},\left\{Q_{n} ; n=1,2,3, \cdots\right\}$, $\left\{R_{n} ; n=1,2,3, \cdots\right\}$ and $\left\{S_{n} ; n=1,2,3, \cdots\right\}$ be, respectively, sequences of relatively compact subdomains of $\widetilde{D}, D, \widetilde{D}^{\sharp}$ and $D^{\sharp}$ such that

$$
\begin{array}{ll}
P_{n} \Subset P_{n+1}, \quad Q_{n} \Subset Q_{n+1}, & R_{n} \Subset R_{n+1}, \quad S_{n} \Subset S_{n+1}(n \geqq 1), \\
\tilde{D}=\bigcup_{n=1}^{\infty} P_{n}, \quad D=\bigcup_{n=1}^{\infty} Q_{n}, \quad \tilde{D}^{\sharp}=\bigcup_{n=1}^{\infty} R_{n}, \quad D^{\sharp}=\bigcup_{n=1}^{\infty} S_{n}, \\
\mu\left(Q_{n}\right) \subset P_{n}, \quad \lambda\left(S_{n}\right) \subset Q_{n}, \quad \tilde{\lambda}\left(R_{n}\right) \subset P_{n}, \quad \kappa\left(S_{n}\right) \subset R_{n}
\end{array}
$$

and $P_{n}, R_{n}$ are, respectively, analytic polycylinders defined by holomorphic functions in $\tilde{D}$ and $\tilde{D}^{\sharp}$. If we take a suitable subsequence $\left\{\nu_{n} ; n=1,2,3, \cdots\right\}$ of $\{1,2,3, \cdots\}$, the discussion below can be continued. For the sake of brevity we may assume that $\nu_{n}=n$ without losing generality. There exists, respectively, subdomains ' $P_{n},{ }^{\prime} Q_{n},{ }^{\prime} R_{n}$ and ' $S_{n}$ of $\widetilde{D}_{n}, D_{n}, \widetilde{D}_{n}^{\sharp}$ and $D_{n}^{\sharp}$ such that $\tilde{\tau}_{n}, \tau_{n}, \tilde{\tau}_{n}^{\sharp}$ and $\tau_{n}^{\sharp}$ map biholomorphically ${ }^{\prime} P_{n},{ }^{\prime} Q_{n},{ }^{\prime} R_{n}$ and ' $S_{n}$ onto $P_{n}, Q_{n}, R_{n}$ and $S_{n}$ and that

$$
\begin{aligned}
& \tilde{\tau}_{n+1}^{n}\left({ }^{\prime} P_{n}\right) \subset^{\prime} P_{n+1}, \quad \tau_{n+1}^{n}\left({ }^{\prime} Q_{n}\right) \subset^{\prime} Q_{n ! 1}, \quad \tilde{\tau}_{n \mid 1}^{\sharp n}\left({ }^{\prime} R_{n}\right) \subset^{\prime} R_{n \mid 1}, \\
& \tau_{n+1}^{\sharp n}\left({ }^{\prime} S_{n}\right) \subset^{\prime} S_{n+1}, \\
& \mu_{n}\left({ }^{\prime} Q_{n}\right) \subset^{\prime} P_{n}, \quad \lambda_{n}\left({ }^{\prime} S_{n}\right) \subset^{\prime} Q_{n}, \quad \tilde{\lambda}_{n}\left({ }^{\prime} R_{n}\right) \subset^{\prime} P_{n}, \quad \kappa_{n}\left({ }^{\prime} S_{n}\right) \subset^{\prime} R_{n}
\end{aligned}
$$

and the commutativity holds in the following diagram:

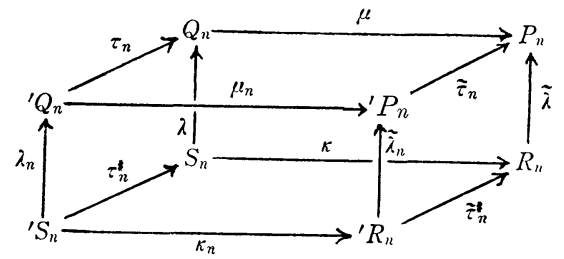

Under this preperation of the notations we shall preceed in the proof of our Lemma. Let $\mathfrak{B}=\left\{V_{i} ; i \in I\right\}$ be any open covering of $D$ and $\left\{f_{i j}\right\}$ be any element of $Z^{1}\left(\mathfrak{B}, \mathfrak{D}^{*}\right)$ such that $\left\{f_{i j} \circ \tau_{n}\right\} \in \mathrm{B}^{1}\left(\tau_{n}^{-1}(\mathfrak{B}), \mathfrak{D}^{*}\right)$ for $n \geqq 1$ where $\tau_{n}^{-1}(\mathfrak{V})=\left\{\tau_{n}^{-1}\left(V_{i}\right) ; i \in I\right\}$ is an open covering of $D_{n}$. There exists $\left\{f_{\imath}^{n}\right\} \in \mathrm{C}^{0}\left(\tau_{n}^{-1}(\mathfrak{B}), \mathfrak{D}^{*}\right)$ such that

$$
f_{\imath j} \circ \tau_{n}=f_{\imath}^{n} / f_{\jmath}^{n}
$$

in $\tau_{n}^{-1}\left(V_{\imath}\right) \cap \tau_{n}^{-1}\left(V_{j}\right) \neq \phi$ for $n \geqq 1$. If we put

$$
f^{n}=f_{2}^{n} / f_{\imath}^{n+1} \circ \tau_{n+1}^{n}
$$

in $\tau_{n}^{-1}\left(V_{\imath}\right)$, then $f^{n}$ is well-defined and belongs to $\mathrm{H}^{0}\left(D_{n}, D^{*}\right)$. Since $\left(\widetilde{D}_{n}, \tilde{\varphi}_{n}\right)$ is the envelope of holomorphy of $\left(D_{n}, \varphi_{n}\right)$, there exists $\tilde{f}^{n} \in \mathrm{H}^{0}\left(\widetilde{D}_{n}, D^{*}\right)$ satisfying

$$
f^{n}=\tilde{f}^{n} \circ \mu_{n}
$$

Then $\log \left(\tilde{f}^{n} \circ \tilde{\lambda}_{n}\right) \in \mathrm{H}^{0}\left(\tilde{D}_{n}^{\sharp}, \mathfrak{D}\right)$ for any fixed branch of logarithmus $(n \geqq 1)$. There holds $\log \left(\tilde{f}^{n} \circ \tilde{\lambda}_{n} \circ\left(\left.\tilde{\tau}_{n}^{*}\right|^{\prime} R_{n}\right)^{-1}\right) \in \mathrm{H}^{0}\left(R_{n}, \mathfrak{D}\right)$ for $n \geqslant 1$ where $\left.\tilde{\tau}_{n}^{\sharp}\right|^{\prime} R_{n}$ is the restriction of $\tilde{\tau}_{n}^{*}$ to 
${ }^{\prime} R_{n}$. Since $R_{n}$ is an analytic polycylinder defined by holomorphic functions in $\tilde{D}^{\ddagger}$, there exists a holomorphic function $\tilde{h}^{n} \in \widetilde{D}^{\sharp}$ such that

$$
\left|\log \left(\tilde{f}^{n} \circ \tilde{\lambda}_{n} \circ\left(\left.\tilde{\tau}_{n}^{\#}\right|^{\prime} R_{n}\right)^{-1}\right)-\tilde{h}^{n}\right|<2^{-n-2}
$$

in $R_{n-1}$ for $n \geqq 2$ from Behnke [1]. We put

$$
H^{n}=\exp \left(\tilde{h}^{n} \circ \kappa\right) \in \mathrm{H}^{0}\left(D^{*}, \mathfrak{D} *\right) .
$$

There holds

$$
\left|\left(f^{n} \circ\left(\left.\tau_{n}\right|^{\prime} Q^{n}\right){ }^{1} \circ \lambda\right) / I^{n}-1\right|<2^{-n}
$$

in $S_{n-1}$ for $n \geq 2$. We put

$$
G^{1}=1, \quad G^{n}=H^{1} H^{2} \cdots H^{n-1} \in H^{0}\left(D^{*}, \mathfrak{D}^{*}\right)
$$

and

$$
F_{\imath}^{n}=\left(f_{\imath}^{n} \circ\left(\left.\tau_{n}\right|^{\prime} Q_{n}\right)^{-1} \circ \lambda\right)\left(G^{n} \in \mathrm{H}^{0}\left(\lambda^{-1}\left(V_{\imath} \cap Q_{n}\right), \mathfrak{D}^{*}\right) .\right.
$$

Then we have $\left\{F_{\imath}^{n}\right\} \in \mathrm{C}^{0}\left(\lambda^{-1}\left(\mathfrak{B} \cap Q_{n}\right), \mathfrak{D}\right)$ where $\lambda^{-1}\left(\mathfrak{B} \cap Q_{n}\right)=\left\{\lambda^{-1}\left(V_{\imath} \cap Q_{n}\right) ; \imath \in I\right\}$ is an open covering of $\lambda^{-1}\left(Q_{n}\right)$. There holds

$$
\left|F_{2}^{n}\right| F_{2}^{n+1}-1 \mid<2^{-n}
$$

in $S_{n-1} \cap \lambda^{-1}\left(V_{1}\right)$. Hence each $F_{i}^{n}$ converges uniformly in any compact subset of $\lambda^{-1}\left(V_{\imath}\right)$ to $F_{i} \in \mathrm{H}^{0}\left(\lambda^{-1}\left(V_{\imath}\right), \mathfrak{D}^{*}\right)$. Since there holds

$$
f_{\imath j} \circ \lambda=F_{i} / F_{j}
$$

in $\lambda^{-1}\left(V_{\imath} \cap V_{j}\right) \neq \phi$, we have proved our lemma.

Corollary of Lemma 1. If $\mathrm{H}^{1}\left(D_{n}, \mathfrak{D}^{*}\right)=0$ for $n \geqq 1$, then we have $\mathrm{H}^{1}\left(D, \mathfrak{D}^{*}\right)=0$.

\section{§ 2. Domains $\boldsymbol{D}$ with $\mathrm{H}^{1}\left(D, \mathfrak{D}^{*}\right)=0$.}

A collection $\left(s=\left\{\left(m_{i}, U_{\imath}, V_{i}\right) ; i \in I\right\}\right.$ is called a multiple Cousm's distribution in a complex space $X$ with essential singularities if the following conditions are satisfied:

(1) $\mathfrak{U}=\left\{U_{i} ; i \in I\right\}$ is an open covering of $X$.

(2) Each connected component of $U_{\imath} \cap U_{j} \cap U_{k}$ contains that of $V_{\imath} \cap V_{j} \cap V_{k}$ for any $U_{\imath} \cap U_{j} \cap U_{k} \neq \phi$.

(3) $m_{i}$ is a single-valued meromorphic function in an open subset $V_{\imath}$ of $U_{\imath}$ for any $i . \quad m_{i} / m_{j}$ can be analytically continued to a function belonging to $\mathrm{H}^{0}\left(U_{2} \cap U_{\jmath}, \mathfrak{D}^{*}\right)$ for any $U_{\imath} \cap U_{\jmath} \neq \phi$.

$\left\{m_{i} / m_{j}\right\}$ defines an element of $Z^{1}\left(\mathfrak{H}, \mathfrak{D}^{*}\right)$ from the condition (2). Its canonical image in $\mathrm{H}^{1}\left(X, \mathfrak{D}^{*}\right)$ is denoted by $\alpha$. A meromorphic function $m$ in $X^{\prime}=\cup_{\imath \in I} V_{2}$ is called a solution of $\mathrm{B}$ if $\mathrm{m} / \mathrm{m}_{i}$ can be analytically continued to a function belonging to $\mathrm{H}^{0}\left(U_{\imath}, \mathfrak{D}^{*}\right)$ for any $i$. Let $X^{\#}$ be a universal covering space of $X$ and $\lambda$ be the canonical mapping of $X^{\#}$ onto $X$. A meromorphic function $M$ in $\lambda^{-1}\left(X^{\prime}\right)$ is called a multiform solution of ( 5 if $M / m_{i} \circ \lambda$ can be analytically continued to a function belonging to $\mathrm{H}^{0}\left(\lambda^{-1}\left(U_{2}\right), \mathfrak{D}^{*}\right)$ for any $i$. For the canonical homomorphism 
$\lambda^{*}: \mathrm{H}^{1}\left(X, \mathfrak{D}^{*}\right) \rightarrow \mathrm{H}^{1}\left(X^{\sharp}, \mathfrak{D}^{*}\right)$, we put ${ }^{*} \alpha=\lambda^{*}(\alpha)$ and ${ }^{*} \mathrm{H}^{1}\left(X, \mathfrak{D}^{*}\right)=\lambda^{*}\left(\mathrm{H}^{1}\left(X, \mathfrak{D}^{*}\right)\right)$.

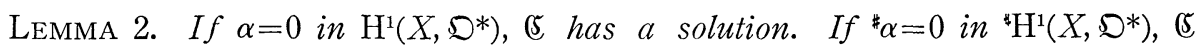
has a multiform solution.

Proof. We shall prove the last half of our Lemma. If ${ }^{*} \alpha=0$ in ${ }^{*} \mathrm{H}^{1}\left(X, \mathfrak{D}^{*}\right)$, $\left\{m_{i} \circ \lambda / m_{j} \circ \lambda\right\}$ defines a coboundary of $\left\{F_{\imath}\right\} \in \mathrm{C}^{0}\left(\lambda^{-1}(\mathfrak{H}), \mathfrak{D}^{*}\right)$ as $\mathrm{H}^{1}\left(\lambda^{-1}(\mathfrak{l}), \mathfrak{D}^{*}\right) \rightarrow \mathrm{H}^{1}\left(X^{\#}, \mathfrak{D}^{*}\right)$ is injective. If we put

$$
M=m_{i} \circ \lambda / F_{i}
$$

in $\lambda^{-1}\left(V_{\imath}\right)$ for any $i \in I, M$ is well-defined and a meromorphic function in $\lambda^{-1}\left(X^{\prime}\right)$ which is a multiform solution of $(5$.

A complex space $X$ is called a Cousin-II-E space (or a multiform Cousin-II-E space) if any multiple Cousin's distribution in $X$ with essential singularities has a solution (or a multiform solution).

Corollary of Lemma 2. If $\mathrm{H}^{1}\left(X, \mathfrak{D}^{*}\right)=0 \quad\left(\right.$ or $\left.\mathrm{H}^{1}\left(X, \mathfrak{D}^{*}\right)=0\right), X$ is a CousinII-E space (or a multiform Cousin-II-E space).

A function $h$ in a set $A$ is called a trace of a function $f$ in the superset $B$ of $A$ if there holds $h=f$ in $A$.

Lemma 3. Let $(D, \varphi)$ be a multiform Cousin-II-E domain over $C^{n},\left(D^{\sharp}, \varphi^{\sharp}\right)$ be the universal covering manifold of $(D, \varphi)$ and $\lambda$ be the canonical mapping of $D^{\sharp}$ onto $D$. Then for any $(n-1)$-dimensional analytic plane $H$ in $C^{n}$ and for any holomorphic function $h$ in $\varphi^{-1}(H), h \circ \lambda$ is a trace of a holomorphic function $f$ in $D^{\sharp}$.

Proof. Without loss of generality we may assume that

$$
H=\left\{\left(z_{1}, z_{2}, \cdots, z_{n}\right) ; z_{1}=0\right\} .
$$

There exists an open neighbourhood $V$ of $\varphi^{-1}(H)$ such that $h$ is a trace of a holomorphic function $h^{\prime}$ in $V$. We can take another open subset $U$ of $D$ such that $\mathfrak{U}=\{U, V\}$ is an open covering of $D$ and $U \cap \varphi^{-1}(H)=\phi$. Then

$$
\left(5=\left\{(1, U, U),\left(\exp \left(h^{\prime} / z_{1} \circ \varphi\right), V, V-\varphi^{-1}(H)\right)\right\}\right.
$$

is a multiple Cousin's distribution in $D$ with essential singularities. Hence there exists a multiform solution $M$ of $\Subset$. We have $M \in \mathrm{H}^{0}\left(D^{\sharp}-\varphi^{\sharp-1}(H), \mathfrak{D}^{*}\right)$. If we start any function element defined by $\log M$ at a point of $D^{\sharp}-\varphi^{\sharp-1}(H)$, it can be not only analytically continued along any curve in $D^{\sharp}-\varphi^{\sharp-1}(H)$ but also meromorphically continued at any point of $\varphi^{\sharp-1}(H)$. Since the fundamental group of $D^{\sharp}$ vanishes, it defines a meromorphic function in $D^{\sharp}$ which we shall denote by the same symbol $\log M$. If we put

$$
f=\left(z_{1} \circ \varphi^{\sharp}\right) \log M,
$$

$f$ is a holomorphic function in $D^{\sharp}$ whose trace in $\varphi^{\sharp-1}(H)$ is $h \circ \lambda$.

Lemma 4. Let $(G, \varphi)$ be a multiform Cousin-II-E domain over $C^{n}$ such that 
$\varphi^{-1}(H)$ is a multiform Cousin-II-E open set over II for any m-dimensional analytic plane $H=\left\{z=\left(z_{1}, z_{2}, \cdots, z_{n}\right) ; z_{\jmath}=c_{\jmath}\left(j=s_{1}, s_{2}, \cdots, s_{n-m}\right)\right\}$ where $m, s_{1}, s_{2}, \cdots$ and $s_{n-m}$ are integers with $1 \leqq m<n, 1 \leqq s_{1}<s_{2}<\cdots<s_{n-m} \leqq n$ and $c$ 's are complex numbers. Then $G$ is a domain of holomorphy.

Proof. We shall prove our Lemma by induction with respect to $n$. For $n=1$ there is nothing to prove from Behnke-Stein [3]. Suppose that our Lemma is valid for all $n \leqq k$ and consider the case $n=k+1$. For any $H \varphi^{-1}(H)$ is an open set of holomorphy over $I I$ from the assumption of our induction. Let $E$ be the set of all boundary point $x^{0}$ of $G$ such that $x^{0}$ is a boundary point of $\varphi^{-1}(H)$ for some $k$ dimensional analytic plane $H=\left\{z ; z_{\jmath}=z_{j}^{0}\right\}$ where $\left(z_{1}^{0}, z_{2}^{0}, \cdots, z_{n}^{0}\right)=\varphi\left(x^{0}\right)$. Then $E$ is dence in $\partial G$. Let $x^{0}$ be a point of $E$ and $I I$ be a $k$-dimensional analytic plane satisfying the above condition for this $x^{0}$. Let $\left(G^{\sharp}, \varphi^{\sharp}\right)$ be the universal covering manifold of $(G, \varphi)$ and $\lambda$ be the canonical mapping of $D^{\sharp}$ onto $D$. Since $\varphi^{-1}(H)$ is an open set of holomorphy, there exists a holomorphic function $h$ in $\varphi^{-1}(H)$ which is unbounded at $x^{0}$. Since $G$ is a multiform Cousin-II-E domain, there exists a holomorphic function $f$ in $G^{\sharp}$ such that

$$
f=h \circ \lambda
$$

in $\varphi^{\sharp-1}(I)$ from Lemma 3. Hence any boundary point of $\left(G^{\sharp}, \varphi^{\sharp}\right)$ belonging to $\lambda^{-1}(E)$ has the frontier property in the sense of Bochner-Martin [4]. Since $E$ is dense in $\partial G$, there exists a holomorphic function $g$ in $G^{\#}$ which is unbounded at each boundary point of $\left(G^{\sharp}, \varphi^{\sharp}\right)$ from [4]. $\left(G^{*}, \varphi^{\sharp}\right)$ is a covering manifold of the domain of holomorphy of $g$. Hence $\left(G^{*}, \varphi^{\sharp}\right)$ is a domain of holomorphy from Oka [13] or Stein [15]. $(G, \varphi)$ is also a domain of holomorphy from Oka [13].

Corollary of Lemma 4. Any multiform Cousin-II-E domain over $C^{2}$ is a domain of holomorphy.

\section{§ 3. Domain exhausted by regular* domains.}

A domain $G$ in $C^{n}$ is called exhausted by regular* domains $G_{p}$ if $G_{p}$ 's are regular* domains in $C^{n}$ such that

$$
G_{p} \Subset G_{p+1}(p=1,2,3, \cdots) \text { and } G=\bigcup_{p=1}^{\infty} G_{p} .
$$

Lemma 5. Let $G$ be a domain in $C^{n}$ exhausted by regular* domains $G_{p}$. Then ${ }^{*} \mathrm{H}^{1}\left(G, \mathfrak{D}^{*}\right)=0$. Moreover for any integers $1 \leqq m<n, 1 \leqq s_{1}<s_{2}<\cdots<s_{n-m} \leqq n$ and for any complex numbers $c_{\jmath}\left(j=s_{1}, s_{2}, \cdots, s_{n-m}\right)$ the intersection $G \cap I I$ of $G$ and $H=\left\{z=\left(z_{1}, z_{2}, \cdots, z_{n}\right) ; z_{j}=c_{j}\left(j=s_{1}, s_{2}, \cdots, s_{n-m}\right)\right\}$ satisfies ${ }^{*} \mathrm{H}^{1}\left(G \cap H, \mathfrak{D}^{*}\right)=0$.

Proof. Since $G_{p}$ is a relatively compact regular* domain, we have $\mathrm{H}^{1}\left(G_{p}, \mathfrak{D} *\right)=0$ for any $p$. From Corollary of Lemma 1 we have $\mathrm{H}^{1}\left(G, \mathfrak{D}^{*}\right)=0$.

Next we shall prove ${ }^{*} \mathrm{H}^{1}\left(G \cap H, \mathfrak{D}^{*}\right)=0$. We may assume that

$$
H=\left\{(z, w)=\left(z_{1}, z_{2}, \cdots, z_{m}, w_{1}, w_{2}, \cdots, w_{n-m}\right) ; w_{j}=0 \quad(j=1,2, \cdots, n-m)\right\} .
$$


There exist $\varepsilon_{p}>0$ and $a_{p}>0$ such that

$$
\begin{gathered}
E_{p}=G_{p} \cap\left\{(z, w) ;\left|z_{j}\right|<a_{p},\left|w_{k}\right|<\varepsilon_{p}(j=1,2, \cdots, m, k=1,2, \cdots, n-m)\right\} \\
\subset\left\{(z, w) ;\left|z_{j}\right|<a_{p},\left|w_{k}\right|<\varepsilon_{p},(z, 0) \in G \cap H,(j=1,2, \cdots, m, k=1,2, \cdots, n-m)\right\}, \\
a_{p}<a_{p+1}(p \geqq 1) \text { and } a_{p} \rightarrow \infty \quad(p \rightarrow \infty), \\
\varepsilon_{p}>\varepsilon_{p+1}(p \geqq 1) \quad \text { and } \quad \varepsilon_{p} \rightarrow 0(p \rightarrow \infty) .
\end{gathered}
$$

Since $G_{p}$ is regular*, we have $\mathrm{H}^{1}\left(E_{p}, \mathfrak{D}^{*}\right)=0$ for any $p$. We put

$$
H_{p}=G_{p} \cap H \cap\left\{(z, 0) ;\left|z_{j}\right|<a_{p}(\jmath=1,2, \cdots, m)\right\} .
$$

Then $G \cap H$ is the limit of monotonously increasing sequence of open sets $H_{p}$ in $H$. Let $\mathfrak{B}=\left\{V_{s} ; s \in S\right\}$ be an open covering of $G \cap H$. We put $V_{s}^{p}=V_{s} \cap H_{p}$ for $s \in S$. Then $\mathfrak{B}_{p}=\left\{V_{s}^{p}\right.$; $\left.s \in S\right\}$ is an open covering of $H_{p}$. We put

$$
U_{s}^{p}=E_{p} \cap\left\{(z, w) ;(z, 0) \in V_{s}\right\}
$$

for $s \in S$. Then $\mathfrak{H}_{p}=\left\{U_{s}^{p}\right.$; s€S $\}$ is an open covering of $E_{p}$. Let $\left\{f_{s t}(z)\right\}$ be an element of $Z^{1}\left(\mathfrak{B}, \mathfrak{D}^{*}\right)$. We put

$$
F_{s t}^{p}(z, w)=f_{s t}(z)
$$

in $U_{s}^{p} \cap U_{t}^{p} \neq \phi$. Then $\left\{F_{s t}^{p}\right\} \in Z^{1}\left(\mathfrak{U}_{p}, \mathfrak{D}^{*}\right)=\mathrm{B}^{1}\left(\mathfrak{U}_{p}, \mathfrak{D}^{*}\right)$ as $\mathrm{H}^{1}\left(\mathfrak{U}_{p}, \mathfrak{D}^{*}\right) \rightarrow \mathrm{H}^{1}\left(E_{p}, \mathfrak{D}^{*}\right)=0$ is injective. There exists $F_{s}^{p} \in \mathrm{H}^{0}\left(U_{s}^{p}, \bigcirc^{*}\right)$ for any $s \in S$ such that

$$
F_{s t}^{p}=F_{s}^{p} / F_{t}^{p}
$$

in $U_{s}^{p} \cap U_{t}^{p} \neq \phi$. If we put

$$
f_{s}^{p}(z)=F_{s}^{p}(z, 0)
$$

in $V_{s}^{p}$ for any $s \in S$, then we have

$$
f_{s t}=f_{s}^{p} / f_{t}^{p}
$$

in $V_{s}^{p} \cap V_{t}^{p} \neq \phi$. Therefore the restriction of $\left\{f_{s t}\right\}$ in any $I I_{p}$ is a coboundary of $\left\{f_{s}^{p}\right\} \in \mathrm{C}^{0}\left(\mathfrak{S}_{p}, \mathfrak{D}^{*}\right)$ for any $p$. From Lemma $1\left\{f_{s t^{\circ}} \lambda\right\} \in \mathrm{B}^{1}\left(\lambda^{-1}(\mathfrak{B}), \mathfrak{D}^{*}\right),\left(G^{\sharp}, \lambda\right)$ being the universal covering manifold of $G$. Thus we have $\mathrm{H}^{1}\left(G \cap H, \mathfrak{D}^{*}\right)=0$.

From Corollary of Lemma 2 and Lemmas 4 and 5 we have

Proposition 1. A domain in $C^{n}$ exhausted by regular* domains is a domain of holomorphy.

\section{§4. Regular* domain with a continuous boundary.}

A boundary point $x^{0}$ of an open set $G$ in $R^{n}$ is called a continuous boundary point of $G$ if there exists a real-valued continuous function $g$ of variables $x_{1}, x_{2}, \cdots, \hat{x}_{\jmath}, \cdots, x_{n}$ in a neighbourhood $V$ of $x^{0}$ such that

$$
G \cap V=\left\{x=\left(x_{1}, x_{2}, \cdots, x_{n}\right) ; x_{\jmath}=g\left(x_{1}, x_{2}, \cdots, \hat{x}_{\jmath}, \cdots, x_{n}\right), x \in V\right\}
$$


for some $j$. A domain $(G, \varphi)$ over a complex manifold is called pseudoconvex at a boundary point $x^{0}$ if there exists an open neighborhood $V$ of $\varphi\left(x^{0}\right)$ such that the connected component of $\varphi^{-1}(V)$ belonging to the filtre defining $x^{0}$ is holomorphically convex. A boundary point $x^{0}$ of a domain $(G, \varphi)$ over a complex manifold is called a simultaneously continuous and locally regular* boundary point of $(G, \varphi)$ if there exists a biholomorphic mapping $\tau$ of an open neighbourhood $V$ of $\varphi\left(x^{0}\right)$ onto a subdomain of a complex Euclidean space such that $\tau \circ \varphi$ maps the connected component $W$ of $\varphi^{-1}(V)$ belonging to the filtre defining $x^{0}$ biholomorphically onto $\tau(\varphi(W))$ and $\tau\left(\varphi\left(x^{0}\right)\right)$ is a continuous boundary point of $\tau(\varphi(W))$ which is a regular* open set. If any boundary point of $(G, \varphi)$ is a simultaneously continuous and locally regular* boundary point, $(G, \varphi)$ is called to have a simultaneously continuous and locally regular* boundary.

Proposition 2. A regular* open set $G$ in $C^{n}$ is pseudoconvex at a continuous boundary point $z^{0}$ of $G$.

Proof. We put $z^{0}=\left(z_{1}^{0}, z_{2}^{0}, \cdots, z_{n}^{0}\right)$. There exists $\varepsilon>0$ and a real-valued continuous function $g$ of variables $z_{1}, z_{2}, \cdots, z_{\jmath-1}, y_{1}, z_{\jmath+1}, \cdots, z_{n}$ in a neighbourhood $V=\left\{z=\left(z_{1}, z_{2}, \cdots, z_{n}\right) ;\left|z_{k}-z_{k}^{0}\right|<\varepsilon(k=1,2, \cdots, n)\right\}$ such that

$$
\partial G \cap V=\left\{z ; x_{j}=g\left(z_{1}, z_{2}, \cdots, z_{\jmath-1}, y_{\jmath}, z_{\jmath+1}, \cdots, z_{n}\right), z \in V\right\}
$$

for some $j$ where $z_{j}=x_{j}+\sqrt{-1} y_{j}$. Then three cases (1), (2) and (3) may occur.

$$
G \cap V=\left\{z ; x_{j}<g\left(z_{1}, z_{2}, \cdots, z_{\jmath-1}, y_{\jmath}, z_{\jmath \mid 1}, z_{n}\right), z \in V\right\} .
$$

For $0 \leqq t<1$ we put

$$
V_{t}=\left\{z ;\left|z_{k}-z_{k}^{0}\right|<(1-t) \varepsilon / 2(k=1,2, \cdots, m)\right\} .
$$

Then we have

$$
\left\{z ;\left(z_{1}, z_{2}, \cdots, z_{\jmath-1}, z_{j}-t \varepsilon / 2, z_{\jmath+1}, \cdots, z_{n}\right) \in V_{t}\right\} \subset V
$$

for $0<t<1$. We put

$$
E_{t}=\left\{z ; x_{j}<g\left(z_{1}, z_{2}, \cdots, z_{\jmath-1}, y_{\jmath}, z_{\jmath+1}, \cdots, z_{n}\right)-t \varepsilon / 2, z \in V_{t}\right\} .
$$

Let $P$ be a relatively compact and simply connected polycylinder in $C^{n}$. $E_{t} \cap P$ is mapped onto

$$
\begin{aligned}
& \left\{w ; u_{j}<g\left(w_{1}, w_{2}, \cdots, w_{j-1}, v_{j}, w_{j+1}, \cdots, w_{n}\right),\left(w_{1}, w_{2}, \cdots, w_{j-1}, w_{j}-t \varepsilon / 2, w_{j+1}, \cdots, w_{n}\right) \in V_{t} \cap P\right\} \\
= & G \cap V \cap\left\{z ;\left(z_{1}, z_{2}, \cdots, z_{\jmath-1}, z_{j}-t \varepsilon / 2, z_{\jmath+1}, \cdots, z_{n}\right) \in V_{t} \cap P\right\}
\end{aligned}
$$

by a biholomorphic mapping $w=\left(w_{1}, w_{2}, \cdots, w_{n}\right)=\gamma(z)$ defined by $w_{k}=z_{k} \quad(k \neq j)$, $w_{j}=z_{j}+t \varepsilon / 2$. Since $\gamma\left(E_{t} \cap P\right)$ is the intersection of $G$ and relatively compact and simply connected polycylinders, we have

$$
\mathrm{H}^{1}\left(E_{t} \cap P, \mathfrak{D}^{*}\right)=\mathrm{H}^{1}\left(r\left(E_{t} \cap P\right), \mathfrak{D}^{*}\right)=0 .
$$

Therefore $E_{t}$ is a regular* open set for $0 \leqq t<1$. Since $E_{0}$ is exhausted by regular* domains $\left\{E_{t} ; 0<t<1\right\}, E_{0}=G \cap V_{0}$ is a domain of holomorphy from Proposition 1 . 
Hence $G$ is pseudoconvex at $z^{0}$.

$$
G \cap V=\left\{z ; x_{\jmath}>g\left(z_{1}, z_{2}, \cdots, z_{\jmath-1}, y_{\jmath}, z_{\jmath+1}, \cdots, z_{n}\right), z \in V\right\} .
$$

In this case the situation is quite similar to the case (1).

$$
G \cap V=\left\{z ; x_{\jmath} \neq g\left(z_{1}, z_{2}, \cdots, z_{\jmath-1}, y_{\jmath}, z_{\jmath+1}, \cdots, z_{n}\right), z \in V\right\} .
$$

Let

$$
G_{1}=\left\{z ; x_{j}<g\left(z_{1}, z_{2}, \cdots, z_{\jmath-1}, y_{\jmath}, z_{\jmath+1}, \cdots, z_{n}\right), z \in V\right\}
$$

and

$$
G_{2}=\left\{z ; x_{\jmath}>g\left(z_{1}, z_{2}, \cdots, z_{\jmath-1}, y_{\jmath}, z_{\jmath+1}, \cdots, z_{n}\right), z \in V\right\} .
$$

From the cases (1) and (2) $G_{1}$ and $G_{2}$ are pseudoconvex at $z^{0}$. Hence $G \cap V=G_{1} \cup G_{2}$ is pseudoconvex at $z^{0}$.

\section{$\S 5$. Domain with a simultaneously continuous and locally regular* boundary.}

Proposition 3. A domain $(G, \varphi)$ over a Stein manifold with a simultaneously continuous and locally regular* boundary is a Slem manifold.

Proof. Let $x^{0}$ be a boundary point of $(G, \varphi)$. From the assumption of our Proposition, there exists a biholomorphic mapping $\tau$ of an open neighbourhood $V$ of $\varphi\left(x^{0}\right)$ onto a domain in a complex Euclidean space such that $\tau \circ \varphi$ maps the connected component $W$ of $\varphi^{-1}(V)$ belonging to the filtre defining $x^{0}$ biholomorphically onto $\tau(\varphi(W))$ and $\tau\left(\varphi\left(x^{0}\right)\right)$ is a continuous boundary point of $\tau(\varphi(W))$ which is a regular* open set. From Proposition $2 \tau(\varphi(W))$ is pseudoconvex at $\tau\left(\varphi\left(x^{0}\right)\right)$. Hence $(G, \varphi)$ is pseudoconvex at $x^{0}$. Since any pseudoconvex domain over a Stein manifold is a Stein manifold from Docquier-Grauert [7], $(G, \varphi)$ is a Stein manifold.

A boundary point $x^{0}$ of a domain $(G, \varphi)$ over a complex manifold is called a locally regular* boundary point of $(G, \varphi)$ if there exists a biholomorphic mapping $\tau$ of an open neighbourhood $V$ of $\varphi\left(x^{0}\right)$ in a complex Euclidean space such that the image $\tau(\varphi(W))$ of the connected component $W$ of $\varphi^{-1}(V)$ belonging to the filtre defining $x^{0}$ by the holomorphic mapping $\tau \circ \varphi$ is a regular* open set. A domain over a complex manifold is called to have a locally regular* boundary if its each boundary point is a locally regular* boundary point. A boundary point $x^{0}$ of a domain $(G, \varphi)$ over an $n$-dimensional complex manifold is called a smooth boundary point of $(G, \varphi)$ if there exists a real-valued continuously differentiable function $g$ in an open neighbourhood $V$ of $\varphi\left(x^{0}\right)$ such that $\sum_{j=1}^{2 n}\left(\partial g / \partial t_{j}\right)^{2} \neq 0$ at $\varphi\left(x^{0}\right)$ for real local coordinates $t_{1}, t_{2}, \cdots, t_{2 n}, \varphi$ maps the connected component $W$ of $\varphi^{-1}(V)$ belonging to the filtre defining $x^{0}$ biholomorphically onto $\varphi(W)$ and there holds

$$
\varphi(W)=\{x ; g(x)<0, x \in V\} .
$$

A boundary point of a domain $(G, \varphi)$ over a Stein manifold which is a smooth 
boundary point of $\left(G^{\prime}, \varphi\right)$ and which is a locally regular* boundary point is a simultaneously continuous and locally regular* boundary point of $(G, \varphi)$ in our sense. But a boundary point of $(G, \varphi)$ which is a continuous boundary point of $(G, \varphi)$ and which is a locally regular* boundary point of $(G, \varphi)$ may not perhaps be a simultaneously continuous and locally regular* boundary point of $(G, \varphi)$ in our sense even if we define a continuous boundary point of $(G, \varphi)$ similarly. A domain over a complex manifold is called to have a smooth boundary if its each boundary point is a smooth boundary point. We have

Corollary of Proposition 3. If a doman $(G, \varphi)$ over a Stein manfold with a smooth boundary has a locally regular* boundary, then $(G, \varphi)$ is a Stein manifold.

Let $G$ be a subdomain of a Stein manifold $S$ with a smooth boundary. If $G$ is not a Stein manifold, there exists a boundary point $x^{0}$ of $G$ which is not a locally regular* boundary point of $G$ from the above Corollary. Let $V$ be any local coordinate neighbourhood of $x^{0}$ and $\tau$ be a biholomorphic mapping of $V$ onto a domain in a complex Euclidean space. Then $\tau(G \cap V)$ is not a regular* open set. Hence there exists a relatively compact and simply connected polycylinder $P$ in $\tau(V)$ such that $\mathrm{H}^{1}\left(\tau(G \cap V) \cap P, \mathfrak{D}^{*}\right) \neq 0$. Since $P$ is a Stein manifold analytically contractible to its each point from Riemann's mapping theorem, $\tau^{-1}(P)$ is also a Stein manifold analytically contractible to its each point. We have

THEOREM 1. Let $G$ be a subdomain of a Stein manifold $S$ with a smooth boundary such that $\mathrm{H}^{1}\left(G \cap D, \mathfrak{D}^{*}\right)=0$ for any subdomain $D$ of $S$ which is a Stein manifold analytically contractible to its each point. Then $G$ is a Stein manifold.

Theorem 2. Let $G$ be a subdomain of a Stem manifold $S$ with a smooth boundary. If $G$ is not a Stein manifold, then there exists an arbitrarily small subdomain $D$ of $S$ which is analytically contractible to th each point such that $\mathrm{H}^{1}\left(G \cap D, D^{*}\right) \neq 0$.

If we do not assume the smoothness of the boundary, we only have

THEOREM 3. If a subdomain $G$ of a Stein manifold $S$ can be exhausted by subdomains $G_{p}$ which satisfy $\mathrm{H}^{1}\left(G_{p} \cap D, \mathfrak{D}^{*}\right)=0$ for any subdomain $D$ of $S$ which is a Stein manifold analytically contractible to its each point, then $G$ is a Stein manifold.

Proof. Of course $G_{p}$ 's satisfy

$$
G_{p} \Subset G_{p_{i 1}}(p \geqq 1), \quad G=\bigcup_{p=1}^{\infty} G_{p} .
$$

Let $x^{0}$ be a boundary point of $G$. We consider a biholomorphic mapping $\tau$ of an open neighbourhood $V$ of $x^{0}$ in a complex Euclidean space $C^{n}$. There exists $\varepsilon>0$ such that 


$$
Z=\left\{z=\left(z_{1}, z_{2}, \cdots, z_{n}\right) ;\left|z_{j}-z_{j}^{0}\right|<\varepsilon(j=1,2, \cdots, n)\right\} \Subset \tau(V) .
$$

We put

$$
Z_{p}=\left\{z ;\left|z_{j}-z_{j}^{0}\right|<p \varepsilon /(p+1)(j=1,2, \cdots, n)\right\}, \quad E_{p}=\tau\left(G_{p} \cap V\right) \cap Z_{p}
$$

for $p \geqq 1$. Let $P$ be a relatively compact and simply connected polycylinder in $C^{n}$. Then we have

$$
\tau^{-1}\left(E_{p} \cap P\right)=G_{p} \cap \tau^{-1}\left(Z_{p} \cap P\right) .
$$

Since each connected component of $\tau^{-1}\left(Z_{p} \cap I^{\prime}\right)$ is a Stein manifold analytically contractible to its each point, we have

$$
\mathrm{H}^{1}\left(E_{p} \cap P, \mathfrak{D}^{*}\right)=\mathrm{H}^{1}\left(\tau^{-1}\left(E_{p} \cap P\right), \mathfrak{D}^{*}\right)=0 .
$$

Therefore $E_{p}$ is a regular* open set for any $p \geqq 1$. Since $E=\tau(G \cap V) \cap Z$ is cxhausted by regular* open sets $E_{p}, E$ is a domain of holomorphy from Proposition 1. Hence $G$ is pseudoconvex at its each boundary point and is a Stein manifold from Docquier-Grauert [7].

\section{REFERENCLS}

[1] Bennke, H., Généralisation du théorème de Runge pour les fonctions multiformes des variables complexes. Coll. sur les fonct. des plus. var. Bruxelles (1953).

[2] BehnKe, II., UND K. Stein, Analytische Funktionen mehrerer Veränderlichen zur vorgegebenen Null- und Pollstellenflächen. Jber. Deut. Math. Verein. 47 (1937), $177-192$.

[ 3 ] Behnke, H., Und K. Stein, Entwicklung analytischer Funktionen auf Riemannschen Flächen. Math. Ann. 120 (1948), 430-461.

[4] Bochner, S., and W. T. Martin, Several complex variables. Princeton Univ. Press (1948).

15] Cartan, H., Les problèmes de Poincaré el cle Cousin pour les fonctions de plusieurs variables complexes. C. R. Paris 199 (1934), 1284-1287.

[6] Cartan, H., Sur les premières problèmes des Cousin. C. R. Paris 207 (1938), $558-560$.

[ 7 ] Docquier, F., Und H. Grauert, Levisches Problem und Rungescher Satz für Teilgebiete Steinscher Mannigfaltigkeit. Math. Ann. 140 (1960), 94-123.

[8] Kajiwara, J., On the limit of a monotonous sequence of Cousin's domains. J. Math. Soc. Japan 17 (1965), 36-46.

[ 9 ] Kajiwara, J., On Thullen's example of a Cousin-II domain. Sci. Rep. Kanazawa Univ. 9 (1964), 1-8.

[10] Kajiwara, J., Some characterizations of Stein manifold through the notion of locally regular boundary points. Kōdai Math. Sem. Rep. 16 (1964), 191-198.

[11] Kajiwara, J., Note on a Cousin-II domain over $C^{2}$. Kōdai Math. Sem. Rep. 17 (1965), 44-47.

[12] ОкА, K., Sur les fonctions analytiques de plusieurs variables: II Domaines d'holomorphie. J. Sci. Hiroshima Univ. 7 (1937), 115-130.

[13] ОкА, K., Ibid.: IX Domaines finis sans point critique intérieur. Jap. J. Math. 23 (1953), 97-155. 
[14] Serre, J. P., Quelques problèmes globaux relatifs aux variété de Stein. Coll. fonct. plus. var. Bruxelles (1953), 57-68.

[15] SteIN, K., Überlagerungen holomorph-vollständiger komplexer Räume. Arch. Math. 7 (1956), 354-361.

[16] Thullen, P., Sur les deuxième problème de Cousin. C. R. Paris 200 (1935). 720721.

College of General Education, Nagoya University. 